\title{
Long-term follow-up studies of Gamma Knife surgery for patients with neurofibromatosis Type 2
}

\author{
Clinical article
}

\author{
Shibin Sun, M.D., ANd Ali Liu, M.D. \\ Gamma Knife Center, Beijing Neurosurgical Institute, Tiantan Hospital, Capital University of Medical \\ Sciences, Beijing, China
}

\begin{abstract}
Object. The aim of this study was to evaluate long-term clinical outcomes after Gamma Knife surgery (GKS) for patients with neurofibromatosis Type 2 (NF2) and the role of GKS in the management of NF2.

Methods. From December 1994 through December 2008, a total of 46 patients (21 male, 25 female) with NF2 underwent GKS and follow-up evaluation for at least 5 years at the Gamma Knife Center of the Beijing Neurosurgical Institute. GKS was performed using the Leksell Gamma Knife Models B and C. The mean age of the patients was 30 years (range 13-59 years). A family history of NF2 was found for 9 (20\%) patients. The NF2 phenotype was thought to be Wishart for 20 (44\%) and Feiling-Gardner for $26(56 \%)$ patients. Among these 46 patients, GKS was performed to treat 195 tumors (73 vestibular schwannomas and 122 other tumors including other schwannomas and meningiomas). For vestibular schwannomas, the mean volume was $5.1 \mathrm{~cm}^{3}$ (median $3.6 \mathrm{~cm}^{3}$, range $0.3-27.3 \mathrm{~cm}^{3}$ ), the mean margin dose was 12.9 Gy (range 10-14 Gy), and the mean maximum dose was 27.3 Gy (range 16.2-40 Gy). For other tumors, the mean volume was $1.7 \mathrm{~cm}^{3}\left(\right.$ range $\left.0.3-5.5 \mathrm{~cm}^{3}\right)$, the mean margin dose was $13.3 \mathrm{~Gy}$ (range 11-14 Gy), and the mean maximum dose was 26.0 Gy (range 18.0-30.4 Gy). The median duration of follow-up was 109 months (range 8-195 months).

Results. For the 73 vestibular schwannomas that underwent GKS, the latest follow-up MR images demonstrated regression of $30(41 \%)$ tumors, stable size for 31 (43\%) tumors, and enlargement of $12(16 \%)$ tumors. The total rate of tumor control for bilateral vestibular schwannomas in patients with NF2 was $84 \%$. Of the 122 other types of tumors that underwent GKS, $103(85 \%)$ showed no tumor enlargement. The rate of serviceable hearing preservation after GKS was $31.9 \%(15 / 47)$. The actuarial rates for hearing preservation at 3 years, 5 years, 10 years, and 15 years were $98 \%, 93 \%, 44 \%$, and $17 \%$, respectively. Of the 46 patients, $22(48 \%)$ became completely bilaterally deaf, $17(37 \%)$ retained unilateral serviceable hearing, and 7 (15\%) retained bilateral serviceable hearing. The mean history of the disease course was 12 years (range 5-38 years).

Conclusions. GKS was confirmed to provide long-term local tumor control for small- to medium-sized vestibular schwannomas and other types of tumors, although vestibular schwannomas in patients with NF2 responded less well than did unilateral sporadic vestibular schwannomas. Phenotype is the most strongly predictive factor of final outcome after GKS for patients with NF2. The risk for loss of hearing is high, whereas the risk for other cranial nerve complications is low.
\end{abstract}

(http://thejns.org/doi/abs/10.3171/2014.8.GKS141503)

\section{Key Words - neurofibromatosis Type 2 • Gamma Knife surgery • long-term follow-up studies • stereotactic radiosurgery}

$\mathrm{N}$ EUROFibromatosis Type 2 (NF2) is an autosomal dominant genetic disorder; the symptoms of neurocutaneous syndrome are caused by mutations on chromosome 22q12.,13,17 Comprehensive management of patients with NF2 remains a challenge. The hallmark and main diagnostic criteria for this disease are the presence of bilateral vestibular schwannomas, with or without a family history of NF2, plus unilateral vestibular schwannomas or any 2 of the following: meningioma, glioma,

Abbreviations used in this paper: GKS = Gamma Knife surgery; $\mathrm{NF} 2=$ neurofibromatosis type 2 . neurofibroma, schwannoma, and posterior subcapsular lenticular opacities. ${ }^{5}$ Incidence of this disease is about 1 in $40,000 .{ }^{4}$ Increasingly, more articles ${ }^{7-12,14-16}$ about the clinical outcomes after Gamma Knife surgery (GKS) for vestibular schwannomas in patients with NF2 have led to growing acceptance of this approach as a safe and effective treatment modality for the combined management of NF2 cases. However, the clinical effects of GKS for patients with vestibular schwannomas associated with NF2 are worse than those for patients with sporadic vestibular schwannomas. To evaluate the long-term clinical outcomes of GKS for patients with NF2, we performed a retrospective study of NF2 patients who had received treatment dur- 
ing 1994-2008 at the Gamma Knife Center of the Beijing Neurosurgical Institute and had been potentially available for 5 or more years of follow-up evaluation.

\section{Methods}

\section{Patient Demographics}

During December 1994-December 2008, a total of 46 patients (21 male and 25 female) with NF2 and bilateral vestibular schwannomas underwent GKS and followup evaluations for least 5 years after GKS. The mean age of the patients was 30 years (range 13-59 years); 12 patients were younger than 20 years. A family history of NF2 was found for 9 (20\%) patients; 2 families accounted for 5 of the 9 patients. Of these 46 patients, 13 (28\%) had only bilateral vestibular schwannomas; the other 33 had bilateral vestibular schwannomas in combination with other central tumors: 12 (26\%) combined with multiple schwannomas, 5 (11\%) combined with multiple meningiomas, and $16(35 \%)$ combined with multiple schwannomas and meningiomas. In general, NF2 was divided into 2 types according to phenotype ${ }^{6}$ : Wishart phenotype NF2 is characterized by rapidly progressing multiple cerebral and spinal lesions in patients younger than 20 years, and Feiling-Gardner phenotype NF2 is characterized by slowly progressing single central tumors in patients older than 20 years. In our cohort, 20 (44\%) patients were thought to have Wishart phenotype and 26 (56\%) Feiling-Gardner phenotype NF2.

\section{Radiosurgical Parameters}

From 1994 until 2000, a Leksell Gamma Knife Model B (Elekta AB) was used; from 2000 on, Model C was used. Before May 1996, the Kula planning system (Elekta AB) was used; thereafter, Leksell GammaPlan software (Elekta AB) was used. The 46 patients studied underwent GKS for a total of 195 tumors (73 vestibular schwannomas and 122 other tumors including other schwannomas and meningiomas). The mean number of GKS-treated tumors for each patient was 4 (range 1-17). In this series, 16 patients underwent $1 \mathrm{GKS}$ procedure, 21 patients underwent 2 GKS procedures, 7 patients underwent 3 GKS procedures, and 2 patients underwent 4 GKS procedures. For vestibular schwannomas, the mean volume was 5.1 $\mathrm{cm}^{3}$ (median $3.6 \mathrm{~cm}^{3}$, range $0.3-27.3 \mathrm{~cm}^{3}$ ), the mean margin dose was 12.9 Gy (10-14 Gy), the mean maximum dose was $27.3 \mathrm{~Gy}(16.2-40 \mathrm{~Gy})$, and the mean isodose line was $49 \%$. For other tumors, the mean volume was $1.7 \mathrm{~cm}^{3}$ (range $0.3-5.5 \mathrm{~cm}^{3}$ ), the mean margin dose was 13.3 Gy (11-14 Gy), the mean maximum dose was 26.0 Gy (18.0-30.4 Gy), and the mean isodose line was $51 \%$.

\section{Treatment Modality for Vestibular Schwannomas in} Patients With NF2

Treatment modalities for bilateral vestibular schwannomas in patients with NF2 are shown in Table 1. Bilateral GKS was performed as the primary treatment for vestibular schwannomas in 17 patients; unilateral GKS was performed as the primary treatment for vestibular
TABLE 1: Treatment modalities used for bilateral vestibular schwannomas in 46 patients with NF2*

\begin{tabular}{lc}
\hline \multicolumn{1}{c}{ Treatment } & No. of Patients \\
\hline bilat GKS & 17 \\
unilat GKS \& contralat MS+GKS & 11 \\
unilat GKS \& contralat MS & 8 \\
unilat GKS \& contralat WS & 4 \\
bilat MS+GKS & 2 \\
unilat MS+GKS and contralat WS & 2 \\
bilat WS & 2 \\
\hline
\end{tabular}

* MS = microsurgery; VS = vestibular schwannoma; WS = wait and see (observation).

schwannomas and as the secondary treatment for contralateral postoperative residual or recurrent vestibular schwannomas in 11 patients; unilateral GKS was performed as the primary treatment for vestibular schwannomas and microsurgery was performed for the contralateral vestibular schwannoma in 8 patients; unilateral GKS was performed as the primary treatment for vestibular schwannomas and contralateral vestibular schwannomas underwent observation (wait and see) in 4 patients; bilateral GKS was performed as the secondary treatment for postoperative vestibular schwannomas in 2 patients; unilateral GKS was performed for postoperative vestibular schwannomas and contralateral vestibular schwannomas underwent observation in 2 patients; and bilateral vestibular schwannomas underwent observation in 2 patients. Of 92 vestibular schwannomas in these 46 patients, GKS was performed as the primary treatment for 57 and as the secondary treatment (postoperatively) for 17 ; microsurgery was performed for 8 vestibular schwannomas, and 10 underwent observation.

\section{Follow-Up Evaluations}

For all patients in the series, all post-GKS followup imaging was conducted by MRI. Hearing and facial symptoms were assessed at baseline and during follow-up according to the Gardner-Robertson classification and the House-Brackmann grading system, respectively. Tumor responses on MR images after GKS were classified into 3 categories: tumor regression, stable tumor, or tumor enlargement. Tumor regression was defined as post-GKS tumor volume $10 \%$ less than pre-GKS tumor volume or tumor shrinkage of $2 \mathrm{~mm}$ or more in 1 axis. Stable tumor was defined as post-GKS tumor volume change within $10 \%$ or less than a $2-\mathrm{mm}$ change in 1 axis. Tumor enlargement was defined as post-GKS tumor volume $10 \%$ greater than pre-GKS tumor volume or growth in 1 axis of $2 \mathrm{~mm}$ or more.

\section{Statistical Analyses}

The Kaplan-Meier product-limit method was used to calculate actuarial rates of vestibular schwannoma control, progression-free survival for patients with NF2, and preservation of serviceable hearing. The correlations of potential prognostic factors with progression-free surviv- 


\section{Long-term follow-up after GKS for patients with NF2}

al of NF2 patients and hearing preservation outcome were analyzed for the following: patient sex (male vs female), patient age ( $<20$ years vs $\geq 20$ years), family history of NF2 (no vs yes), phenotype (Wishart vs Feiling-Gardner), type of tumors (bilateral vestibular schwannomas only vs combined with other schwannomas and/or meningiomas), and treatment modality (GKS only vs GKS plus operation or observation). The correlation of potential prognostic factors with hearing preservation and tumor control for 73 vestibular schwannomas was analyzed for treatment modality (GKS only vs GKS plus operation or observation) and tumor volume group ( $<5 \mathrm{~cm}^{3}$ vs $5-10 \mathrm{~cm}^{3}$ vs $\geq$ $10 \mathrm{~cm}^{3}$ ). Univariate analysis was performed on KaplanMeier plots by using the log-rank statistic; a $\mathrm{p}$ value < 0.05 was considered significant. Multivariate analyses were performed by using the Cox regression model ( $95 \%$ confidence intervals). Standard statistical processing software (SPSS version 17.0; SPSS, Inc.) was used.

\section{Results}

\section{Tumor Control}

The median duration of follow-up was 109 months (range 8-195 months). Of the 73 vestibular schwannomas that underwent GKS, the latest follow-up MRI studies demonstrated tumor regression for $30(41 \%)$ tumors, stable tumor for 31 (43\%), and tumor enlargement for 12 $(16 \%)$ tumors. The total rate of tumor control for bilateral vestibular schwannomas in patients with NF2 was $84 \%$. Actuarial rates for local control of vestibular schwannomas were 87 and $41 \%$ at 5 and 10 years, respectively. Of 122 other types of tumors, 103 (85\%) tumors showed no enlargement.

\section{Outcome of Hearing Preservation and Other Cranial Nerve Functions}

Of the 46 NF2 patients (92 vestibular schwannomas) in this cohort, hearing was serviceable (Gardner-Robertson Grades 1 and 2) for 34 patients with 56 vestibular schwannomas that had not undergone surgery before GKS. Before GKS, loss of serviceable hearing was found for 1 patient with bilateral vestibular schwannomas and no previous surgery and for 34 patients with vestibular schwannomas and previous surgery. Up to the last followup evaluation, 9 vestibular schwannomas in 7 patients underwent long-term observation (mean 7.7 years, range 5-10 years), and the rate of serviceable hearing preservation was $33.3 \%$ (3/9); GKS was the primary treatment and hearing level follow-up was completed for patients with 47 vestibular schwannomas, and the rate of serviceable hearing preservation was $31.9 \%$ (15/47); and microsurgery was the primary treatment for 25 vestibular schwannomas, and the rate of serviceable hearing preservation before GKS was nearly $0 \%$. The mean interval of serviceable hearing loss after GKS was about 7 years (range 1-11 years). The actuarial rates for hearing preservation after 3 years, 5 years, 10 years, and 15 years after primary GKS are $98 \%, 93 \%, 44 \%$, and $17 \%$, respectively.

Of the 46 patients in this cohort, $22(48 \%)$ patients became completely bilaterally deaf, 17 (37\%) patients re- tained unilateral serviceable hearing, and 7 (15\%) patients retained bilateral serviceable hearing. Of the 73 vestibular schwannomas, the rate of transient or permanent trigeminal neuropathies was $8 \%$, and the rate of transient or permanent facial paralysis was $5 \%$.

\section{Disease Course}

During the long-term follow-up period, 14 (30\%) patients died or became severely disabled, $29(63 \%)$ patients experienced tumor progression, and 17 (37\%) patients had stable tumors. The disease course was defined as the time of definitive diagnosis to the time of the last follow-up evaluation. The mean history of the disease course was 12 years (range 5-38 years). Of the 46 patients in this group, 5 patients with multiple meningiomas only were all older patients with Feiling-Gardner phenotype NF2 and $8(67 \%)$ of 12 with multiple schwannomas only were juvenile patients with Wishart phenotype NF2. So in our experience, schwannomas tended to develop more in younger patients with NF2 and meningiomas tended to develop more in older patients with NF2. We noted that the disease course in young patients with NF2 progressed rapidly, especially in those with Wishart phenotype NF2 (Fig. 1). Of the 14 patients who died or were severely disabled, 12 patients were thought to have Wishart phenotype NF2, and many schwannomas, neurofibromas, and meningiomas developed throughout the nervous system ( $>50$ tumors in 1 patient with NF2) during a mean disease course of 13 years (range 5-21 years).

Factors Associated With Tumor Progression-Free Survival and Hearing Preservation

According to univariate analysis (performed on Kaplan-Meier plots using the log-rank statistic), $p$ values for association with progression-free survival were as follows: patient sex $(p=0.219)$, patient age $(p=0.016)$, family history of NF2 ( $p=0.203), N F 2$ phenotype $(p=$ $0.011)$, tumor types $(p=0.034)$, and treatment modality ( $p$ $=0.577$ ). The factors identified by univariate analysis as being significantly associated with progression-free survival were patient age, NF2 phenotype, and tumor types. According to multivariate analysis (Cox regression), $\mathrm{p}$ values for association with progression-free survival were as follows: patient sex $(p=0.087)$, patient age $(p=0.475)$, family history of NF2 $(\mathrm{p}=0.127), \mathrm{NF} 2$ phenotype $(\mathrm{p}=$ $0.016)$, tumor types $(p=0.107)$, and treatment modality ( $p$ $=0.946$ ). The only factor identified by multivariate analysis as being significantly associated with progression-free survival was NF2 phenotype.

According to univariate analysis (performed on Kaplan-Meier plots using the log-rank statistic), $p$ values for association with hearing preservation were as follows: patient sex $(\mathrm{p}=0.407)$, patient age $(\mathrm{p}=0.772)$, family history $(p=0.931), N F 2$ phenotype $(p=0.177)$, and treatment modality $(\mathrm{p}=0.418)$. According to multivariate analysis (using Cox regression), $\mathrm{p}$ values for association with hearing preservation were as follows: patient $\operatorname{sex}(p=0.232)$, patient age $(p=0.391)$, family history of NF2 $(p=0.285)$, NF2 phenotype $(\mathrm{p}=0.679)$, and treatment modality $(\mathrm{p}=$ 0.280 ). Neither univariate nor multivariate analyses iden- 

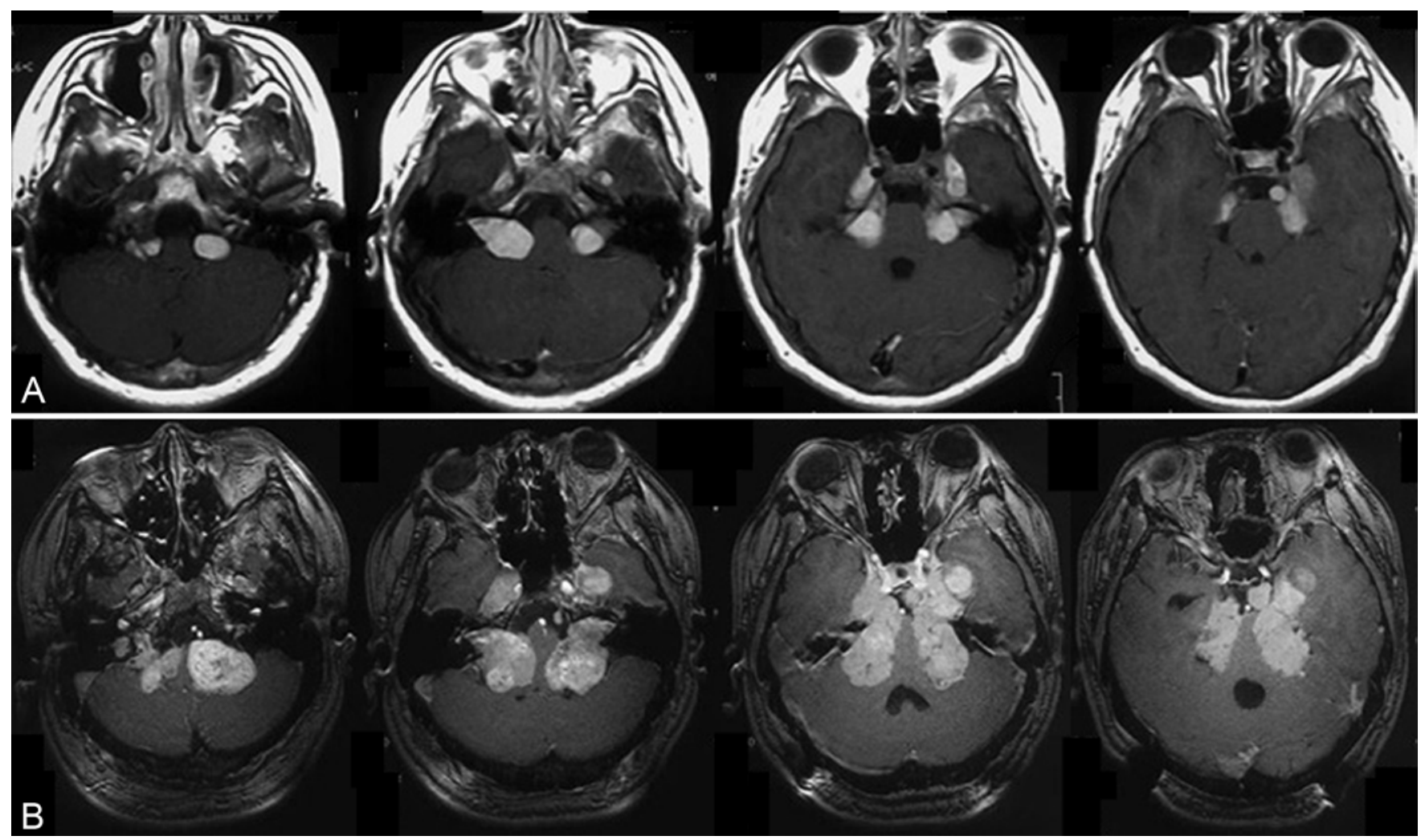

FIG. 1. Serial MR images showing rapid tumor growth 5 years after observation (wait-and-see policy). A: A 16-year-old male patient with multiple schwannomas and cervical spinal ependymoma (treated surgically), thought to have Wishart-phenotype NF2, chose a conservative wait-and-see treatment strategy. B: After 5 years, follow-up MR images demonstrated tumor progression with complete loss of hearing and vision. GKS was then performed for schwannomas on 1 side. During the 5-year follow-up period, the patient became severely disabled and refused follow-up MRI. The disease course was 17 years.

tified a significant factor associated with the outcome of hearing preservation.

Of 73 vestibular schwannomas treated by GKS, according to univariate analysis (performed on KaplanMeier plots using the log-rank statistic), $\mathrm{p}$ values for association with hearing preservation were as follows: treatment modality $(\mathrm{p}=0.724)$ and volume group $(\mathrm{p}=$ 0.569 ). According to univariate analysis (performed on Kaplan-Meier plots by using the log-rank statistic), $\mathrm{p}$ values for association with tumor control were as follows: treatment modality $(\mathrm{p}=0.729)$ and volume group $(\mathrm{p}=$ 0.489 ). The factors of treatment modality and tumor volume size seemed to have no significant effect on hearing preservation and tumor control in NFS patients with vestibular schwannomas.

\section{Discussion}

\section{Tumor Control and Factors Associated With GKS}

With the achievement of GKS treatment of sporadic vestibular schwannomas, more and more clinicians have been interested in long-term clinical results after GKS for vestibular schwannomas in patients with NF2. Recently reported clinical outcomes are listed in Table 2.8-10,14,15 Among patients in our series, the actuarial 5-year and 10year rates of tumor control were $87 \%$ and $41 \%$, respectively; the rate for long-term tumor control was lower than that found by Mathieu et al. ${ }^{9}$ ( $85 \%$ and $81 \%$ at 5 and 10 years, respectively) and Mallory et al. ${ }^{8}(85 \%$ and $80 \%$ at 5 and 10 years, respectively). According to the results presented in Table 2, we note that GKS might provide relatively high rates of long-term control of vestibular schwannomas in patients with NF2 (50\%-87.5\%); these rates are lower than those for sporadic vestibular schwannomas. There was also no evident difference between tumor control rates previously reported ${ }^{7-12,14-16}$ and those from our study (both at prescribed peripheral dose of 12-14 Gy). Thus, there is no doubt that GKS can provide long-term arrest of local tumor growth for vestibular schwannomas in patients with NF2. According to our study and the other studies mentioned above, ${ }^{7-12,14-16}$ the rates of tumor control after GKS for vestibular schwannomas in patients with NF2 are obviously lower than those for sporadic vestibular schwannomas. Consequently, the role of GKS in the management of vestibular schwannomas in patients with NF2 and of sporadic vestibular schwannomas differs to some extent. GKS can benefit patients with sporadic vestibular schwannomas because of the high rate of control of small- to medium-sized vestibular schwannomas, in the absence of other central tumors. But for patients with NF2, especially the Wishart phenotype, GKS played only a limited role in the comprehensive management because of the relatively low rate of tumor progression-free survival and the rapid growth of many other tumors.

From Fig. 2, we concluded that the actuarial progres- 


\section{Long-term follow-up after GKS for patients with NF2}

TABLE 2: Comparison of studies of GKS for vestibular schwannomas in patients with NF2*

\begin{tabular}{lccccc}
\hline \multicolumn{1}{c}{ Authors \& Year } & $\begin{array}{c}\text { No. of Patients } \\
\text { (no. of VSs) }\end{array}$ & $\begin{array}{c}\text { Median Margin } \\
\text { Dose in Gy (range) }\end{array}$ & $\begin{array}{c}\text { Median FU in Mos } \\
\text { (range) }\end{array}$ & $\begin{array}{c}\text { Hearing Preservation } \\
\text { Rate (\%) }\end{array}$ & $\begin{array}{c}\text { Tumor Control } \\
\text { Rate (\%) }\end{array}$ \\
\hline Rowe et al., 2003 & $96(122)$ & $15.2(10-25)$ & $38(4-154)$ & 40 & 50 \\
Mathieu et al., 2007 & $62(74)$ & $14(11-20)$ & 53 & 42 & 83 \\
Phi et al., 2009 & $30(36)$ & $12.1(8-14)$ & $36.5(4-99)$ & 33 & 70 \\
Sharma et al., 2010 & $30(54)$ & $12(10-15)$ & 26.6 & 66.7 & 87.5 \\
Mallory et al., 2014 & $26(32)$ & $14(12-20)$ & $91(12-262)$ & 25 & 84 \\
Sun \& Liu, 2014 (present & $46(73)$ & $12.9(10-14)$ & $109(8-195)$ & 31.9 & 84 \\
$\quad$ study) & & & & &
\end{tabular}

sion-free survival rate after GKS for patients with Wishart phenotype NF2 was less than that for patients with Feiling-Gardner phenotype NF2, and the reason is the rapid development of the disease course in patients with Wishart phenotype NF2. In our experience, phenotype was the only factor that significantly affected progression-free survival according to both univariate and multivariate analyses. Above all, we noted that NF2 phenotype remained the most significant prognostic factor for progression-free survival of NF2 patients. In fact, patient age and type of tumor were both associated with phenotype classification. Mathieu et al..$^{9}$ and Phi et al. ${ }^{10}$ found younger patient age to be a predictor of poor tumor control. It is known that specific mutations in the NF2 gene are associated with younger age at disease onset and with overall severity of the disease. Phi et al. also hold the same view as found in our study, that these genotype-phenotype correlations might also extend to treatment responses. In addition, Mathieu et al. noted that tumor volume was a significant predictor of local control.

\section{Preservation of Serviceable Hearing}

Among the 46 patients, 7 patients with 9 vestibu-

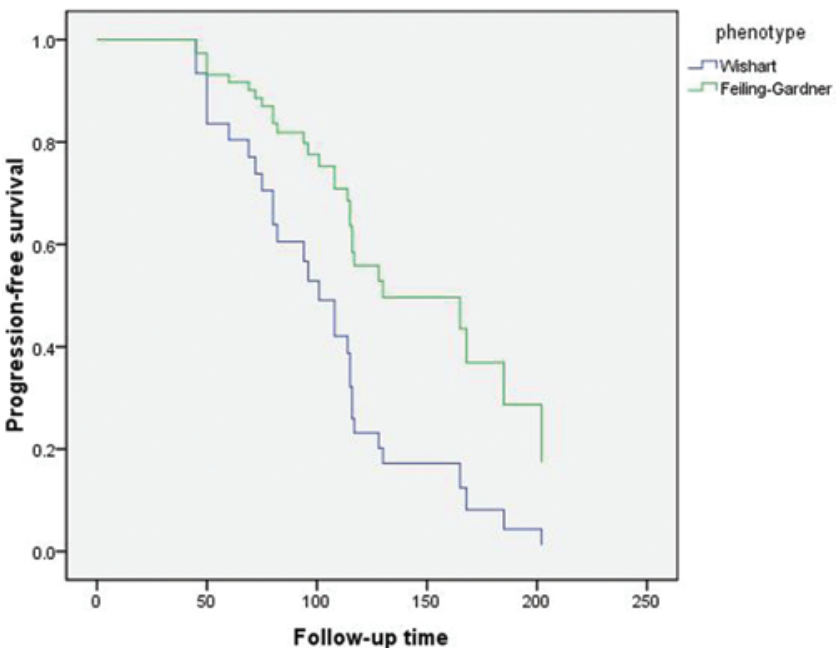

Fig. 2. Kaplan-Meier plot illustrating the significant effect of NF2 phenotype on progression-free survival after GKS. After GKS, the actuarial progression-free survival time for Wishart-phenotype NF2 was less than that for Feiling-Gardner-phenotype NF2. lar schwannomas underwent observation during a mean long-term follow-up period of 8 years (range 7-10 years); 1 patient with a vestibular schwannoma was excluded from analysis because of loss of serviceable hearing before GKS. The hearing of all patients with these 9 vestibular schwannomas tended to deteriorate, and 3 patients lost their serviceable hearing. Serviceable hearing is more likely to be retained in a patient in whom the disease course is indolent. During a 7-year period of clinical observation without GKS, bilateral deafness developed in 1 young female patient; follow-up MRI demonstrated that her bilateral vestibular schwannomas remained stable in size. We inferred that the reason for her deafness was that the tumor cells might have infiltrated and engulfed cranial nerve VIII and the cochlea.

Data from our study and others ${ }^{8-10,14,15}$ show that GKS could provide relatively long-term hearing preservation for $25.0 \%-66.7 \%$ of NF2 patients, which is less than might be expected for hearing preservation among patients with GKS-treated sporadic vestibular schwannomas. According to univariate and multivariate analyses, no significant factors (such as tumor size or treatment modality) were found to be associated with hearing preservation in this group. The prescription margin dose range of GKS for vestibular schwannomas in patients with NF2 was the same as that for sporadic vestibular schwannomas (12-15 Gy); therefore, we considered that the margin dose and the dose affecting the cochlea were also not the important factors. Mallory et al. ${ }^{8}$ noted that hearing outcomes were poor even when contemporary reduced marginal doses were used.

We found no significant difference in the rate of serviceable hearing preservation between the 9 vestibular schwannomas that underwent long-term observation and the 49 vestibular schwannomas that underwent GKS as the primary treatment ( $33.3 \%$ vs $31.9 \%$ ), so we concluded that the degree of serviceable hearing deterioration resulting from the iatrogenic injury of GKS would not exceed that resulting from natural injury during long-term clinical observation (wait and see). We noted that the rapid progression of disease in patients with NF2 was the most significant predictive factor resulting in poor hearing preservation.

\section{Treatment Strategy}

Of the 9 vestibular schwannomas in 7 patients man- 
aged with observation only, follow-up MRI demonstrated that only 2 vestibular schwannomas remained stable in size (tumor control rate only 22.2\%), whereas the rate of preservation of serviceable hearing was $33.3 \%$. For the group for whom GKS was the initial and definitive treatment, the rate of tumor control was about $84 \%$, and the rate of serviceable hearing preservation was $31.9 \%$. Thus, we conclude that GKS arrested tumor growth enough to interrupt or terminate the natural disease course for some patients with Feiling-Gardner phenotype NF2, although loss of serviceable hearing for most patients with NF2 was inevitable. At the Beijing Neurosurgical Institute, a 53-year-old patient with only bilateral vestibular schwannomas experienced 18 years of progression-free survival after GKS (Fig. 3).

At the Beijing Neurosurgical Institute, the choice of treatment modality for NF2 patients is determined by disease progression, local tumor volume, and a desire to preserve bilateral serviceable hearing. For bilateral vestibular schwannomas, the choice of which vestibular schwannomas should undergo GKS is determined by whether hearing is deteriorating on 1 side or if either vestibular schwannoma shows active growth during the clinical observation period. In our experience, we follow a policy of surgical operation for large-volume tumors with increased intracranial pressure, wait and see (clinical observation) is not recommended, and staged GKS is recommended as the primary treatment for patients in the early stage of

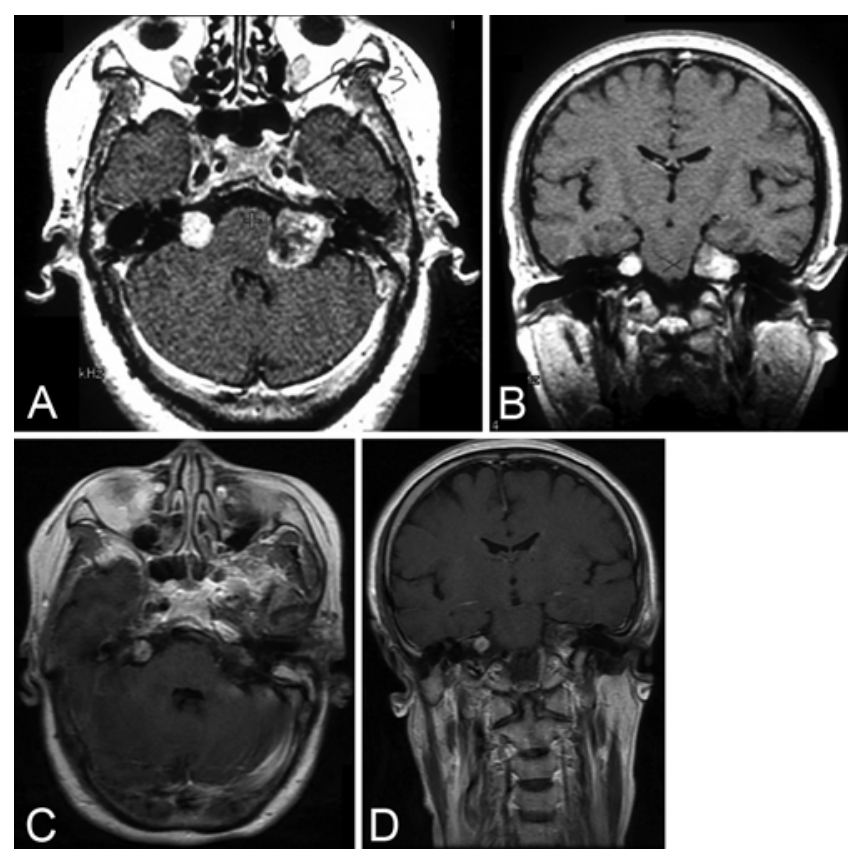

FIG. 3. Serial follow-up MR images showing tumors' shrinkage 18 years after GKS. A: Axial image of GKS localization. B: Coronal image of GKS localization. C: Follow-up axial image 18 years after GKS. D: Follow-up coronal image 18 years after GKS. A 53-yearold female patient with bilateral vestibular schwannomas and thought to have Feiling-Gardner phenotype NF2 underwent GKS as primary treatment. For the left vestibular schwannoma, the peripheral dose was $12.25 \mathrm{~Gy}$ and the central dose was $35 \mathrm{~Gy}$. For the right vestibular schwannoma, the peripheral dose was $12 \mathrm{~Gy}$ and the central dose was 40 Gy. At 8 years after GKS, the patient had lost serviceable hearing on both sides.
NF2 disease. In our experience from this study, we agree with Baser et al. ${ }^{2}$ that the rates of vestibular schwannoma growth in patients with NF2 tend to decrease with increasing patient age. But for younger patients with Wishart phenotype NF2, individual comprehensive management should include not only microsurgery and GKS but also possibly systemic antiangiogenesis target drugs. We have had some experience with a 19 -year-old female patient with multiple schwannomas and meningiomas who was treated with bevacizumab after GKS and microsurgery; follow-up MRI 2 years later showed most tumors to be stable, although bilateral serviceable hearing was nearly lost. Several papers ${ }^{3,11}$ have reported that vascular endothelial growth factor might play a major role in NF2associated vestibular schwannoma growth and that bevacizumab might help preserve serviceable hearing and arrest growth of vestibular schwannomas in some selected NF2 patients.

\section{Conclusions}

In this study, GKS was confirmed to provide longterm local tumor control for small- to medium-sized vestibular schwannomas and other types of tumors, although vestibular schwannomas in patients with NF2 responded less favorably than did unilateral sporadic vestibular schwannomas. Phenotype is the most significant factor for predicting the final outcome after GKS for patients with NF2. The risk for hearing loss is high, whereas the risk for other cranial nerve complications is low.

\section{Disclosure}

The authors report no conflict of interest concerning the materials or methods used in this study or the findings specified in this paper.

Author contributions to the study and manuscript preparation include the following. Conception and design: Sun. Acquisition of data: Sun. Analysis and interpretation of data: Sun. rafting the article: Sun. Critically revising the article: Sun. Reviewed submitted version of manuscript: both authors. Approved the final version of the manuscript on behalf of both authors: Sun. Statistical analysis: Sun. Administrative/technical/material support: Liu. Study supervision: Liu.

\section{References}

1. Baser ME, Kuramoto L, Woods R, Joe H, Friedman JM, Wallace AJ, et al: The location of constitutional neurofibromatosis 2 (NF2) splice site mutations is associated with the severity of NF2. J Med Genet 42:540-546, 2005

2. Baser ME, Makariou EV, Parry DM: Predictors of vestibular schwannoma growth in patients with neurofibromatosis Type 2. J Neurosurg 96:217-222, 2002

3. Eminowicz GK, Raman R, Conibear J, Plowman PN: Bevacizumab treatment for vestibular schwannomas in neurofibromatosis type two: report of two cases, including responses after prior gamma knife and vascular endothelial growth factor inhibition therapy. J Laryngol Otol 126:79-82, 2012

4. Evans DGR, Baser ME, O'Reilly B, Rowe J, Gleeson M, Saeed $\mathrm{S}$, et al: Management of the patient and family with neurofibromatosis 2: a consensus conference statement. Br J Neurosurg 19:5-12, 2005

5. Evans DGR, Huson SM, Donnai D, Neary W, Blair V, Newton $\mathrm{V}$, et al: A genetic study of type 2 neurofibromatosis in 
the United Kingdom. II. Guidelines for genetic counselling. J Med Genet 29:847-852, 1992

6. Evans DGR, Trueman L, Wallace A, Collins S, Strachan T: Genotype/phenotype correlations in type 2 neurofibromatosis (NF2): evidence for more severe disease associated with truncating mutations. J Med Genet 35:450-455, 1998

7. Kida Y, Kobayashi T, Tanaka T, Mori Y: Radiosurgery for bilateral neurinomas associated with neurofibromatosis type 2. Surg Neurol 53:383-390, 2000

8. Mallory GW, Pollock BE, Foote RL, Carlson ML, Driscoll CL, Link MJ: Stereotactic radiosurgery for neurofibromatosis 2-associated vestibular schwannomas: toward dose optimization for tumor control and functional outcomes. Neurosurgery 74:292-301, 2014

9. Mathieu D, Kondziolka D, Flickinger JC, Niranjan A, Williamson R, Martin JJ, et al: Stereotactic radiosurgery for vestibular schwannomas in patients with neurofibromatosis type 2: an analysis of tumor control, complications, and hearing preservation rates. Neurosurgery 60:460-470, 2007

10. Phi JH, Kim DG, Chung HT, Lee J, Paek SH, Jung HW: Radiosurgical treatment of vestibular schwannomas in patients with neurofibromatosis type 2: tumor control and hearing preservation. Cancer 115:390-398, 2009

11. Plotkin SR, Merker VL, Halpin C, Jennings D, McKenna MJ, Harris GJ, et al: Bevacizumab for progressive vestibular schwannoma in neurofibromatosis type 2: a retrospective review of 31 patients. Otol Neurotol 33:1046-1052, 2012

12. Roche PH, Régis J, Pellet W, Thomassin JM, Grégoire R, Dufour $\mathrm{H}$, et al: [Neurofibromatosis type 2. Preliminary results of gamma knife radiosurgery of vestibular schwannomas.] Neurochirurgie 46:339-354, 2000 (Fr)
13. Rouleau GA, Merel P, Lutchman M, Sanson M, Zucman J, Marineau $\mathrm{C}$, et al: Alteration in a new gene encoding a putative membrane-organizing protein causes neuro-fibromatosis type 2. Nature 363:515-521, 1993

14. Rowe JG, Radatz MW, Walton L, Soanes T, Rodgers J, Kemeny AA: Clinical experience with gamma knife stereotactic radiosurgery in the management of vestibular schwannomas secondary to type 2 neurofibromatosis. J Neurol Neurosurg Psychiatry 74:1288-1293, 2003

15. Sharma MS, Singh R, Kale SS, Agrawal D, Sharma BS, Mahapatra AK: Tumor control and hearing preservation after Gamma Knife radiosurgery for vestibular schwannomas in neurofibromatosis type 2. J Neurooncol 98:265-270, 2010

16. Subach BR, Kondziolka D, Lunsford LD, Bissonette DJ, Flickinger JC, Maitz AH: Stereotactic radiosurgery in the management of acoustic neuromas associated with neurofibromatosis Type 2. J Neurosurg 90:815-822, 1999

17. Trofatter JA, MacCollin MM, Rutter JL, Murrell JR, Duyao MP, Parry DM, et al: A novel moesin-, ezrin-, radixin-like gene is a candidate for the neurofibromatosis 2 tumor suppressor. Cell 72:791-800, 1993 (Erratum in Cell 75:826, 1993)

Manuscript submitted July 1, 2014.

Accepted August 5, 2014.

Please include this information when citing this paper: DOI: 10.3171/2014.8.GKS141503

Address correspondence to: Shibin Sun, M.D., Gamma Knife Center, Beijing Neurosurgical Institute, Tiantan Hospital, Capital University of Medical Sciences, No. 6 Chong Wen Qu Tiantanxili, Beijing 100050, China. email: ssbwyl@vip.sina.com. 